\title{
Asymptomatic immunoglobulin light chain amyloidosis (AL) at the time of diagnostic bone marrow biopsy in newly diagnosed patients with multiple myeloma and smoldering myeloma. A series of 144 cases and a review of the literature
}

\author{
Sergio Siragusa - William Morice • Morie A. Gertz $\cdot$ Robert A. Kyle • \\ Philip R. Greipp • John A. Lust • Thomas E. Witzig • Martha Q. Lacy • \\ Steven R. Zeldenrust • S. Vincent Rajkumar • Stephen J. Russell • \\ Suzanne R. Hayman • Francis Buadi • Shaji K. Kumar • David Dingli • \\ Angela Dispenzieri
}

Received: 5 June 2010 / Accepted: 30 June 2010 /Published online: 20 July 2010

(C) Springer-Verlag 2010

\begin{abstract}
The rate of asymptomatic amyloidosis (AL) among patients with newly diagnosed multiple myeloma $(\mathrm{MM})$ or smoldering multiple myeloma (SMM) is unknown. We evaluated number and clinical significance of asymptomatic AL in consecutive MM and SMM patients, not having recognition of symptomatic $\mathrm{AL}$ at the time of their diagnostic bone marrow biopsy. Bone marrow biopsies were stained with Congo red and considered diagnostic for $\mathrm{AL}$ in case of positive Congo red staining with apple-green birefringence. Biopsies from 144 patients were evaluated: 77 had a diagnosis of MM and 67 of SMM. The median age was 59 (range 26-84) years; the median follow-up was 76 months (range 0-216). Immunoglobulin isotypes were 96/144 (67\%), IgG; 23/144 (16\%), IgA; 12/ 144 (8\%), light chain only; 1/77 (1\%), IgD; and biclonal or indeterminate, 12/144 (8\%). Fifty-eight percent (84/144)
\end{abstract}

\footnotetext{
S. Siragusa $(\bowtie)$

Cattedra ed U.O. di Ematologia con trapianto,

Azienda Ospedaliera Universitaria Policlinico di Palermo,

via del Vespro 127 ,

90127 Palermo, Italy

e-mail: sergio.siragusa@unipa.it

W. Morice

Division of Pathology, Mayo Clinic Rochester,

Rochester, MN, USA

M. A. Gertz $\cdot$ R. A. Kyle • P. R. Greipp · J. A. Lust - T. E. Witzig •

M. Q. Lacy · S. R. Zeldenrust - S. V. Rajkumar - S. J. Russell •

S. R. Hayman · F. Buadi · S. K. Kumar • D. Dingli •

A. Dispenzieri

Division of Hematology, Mayo Clinic Rochester,

Rochester, MN, USA
}

were $\mathrm{K}$ restricted. The presence of amyloid was found in two cases $(1 \%, 95 \% \mathrm{CI}-0.6$ to 3.2$)$, one in $\mathrm{MM}$, and one in SMM group, and none had or developed signs or symptoms suggestive of organ involvement by amyloid. Among the 142 other patients without amyloid deposition in their index bone marrow, one $(0.7 \%, 95 \% \mathrm{CI}-0.6$ to 2.0$)$ developed symptomatic AL after 119 months.

Keywords Amyloid · Multiple myeloma . Smoldering myeloma . Asymptomatic .

Bone marrow biopsy

\section{Introduction}

Multiple myeloma (MM), smoldering multiple myeloma (SMM), immunoglobulin light chain amyloidosis (AL), and monoclonal gammopathy of undetermined significance (MGUS) represent a spectrum of plasma cells disorders (PCDs). AL is a rare and serious disorder characterized by the deposition of amyloid fibrils in different tissues with more than $80 \%$ of patients dead within 2 years of diagnosis $[1,2]$

Immunoglobulin light chain amyloidosis may coexist with any of the other PCDs $[2,3]$. It has been reported that approximately $10 \%$ of patients with AL may have MM at the time of diagnosis [2], while only a minority will develop delayed MM [4]. Conversely, it has been reported that up to $30 \%$ of MM patients may have subclinical amyloid deposits (in subcutaneous fat aspirate, bone marrow biopsy, or biopsies of other vital organs) [5-8], 
and in up to $15 \%$ of all MM patients, a clinically overt $\mathrm{AL}$ appears during the course of index MM [3, 5]).

The rate of asymptomatic amyloidosis (asymp-amyloid) in patients with MM or SMM as well as its clinical impact is uncertain. This topic is significant because unrecognized AL can be associated with significant mortality and morbidity. In the case of patients with SMM, no treatment will be directed against the plasma cell clone, potentially allowing for unbridled progression of the AL. Unrecognized AL in patients diagnosed with MM is also significant because of the potential for higher rates of treatment-related morbidity and mortality in patients with occult visceral involvement with AL. However, at the present we deal with discordant information. Some authors found out MMassociated asymp-amyloid in up to $26 \%$ of patients at the time of diagnosis [8]; this was also an independent highrisk prognostic factor for MM patients, thus suggesting need for searching amyloid and need for aggressive treatment. Others believe that AL deposits, even if not rare, are clinically inconsequential and should not influence MM therapy, so active screening is unnecessary $[6,9]$.

Systemic AL can be a devastating disease with an insidious onset, and its early recognition may improve clinical outcomes and patients' management. The objective of the present investigation was therefore that of evaluating the rate of asymp-amyloid in MM and SMM patients at the moment of first diagnostic bone marrow (BM) biopsy.

\section{Design and methods}

\section{Patients}

Study population was selected from the Mayo Clinic Dysproteinemia database (Division of Hematology, Rochester MN, USA) by a computerized search of the medical records of consecutive patients with diagnosis of MM and SM during the period January 1, 1993 to December 31, 2003: Diagnosis was made upon criteria on the International Myeloma Study Group [10]. All patients with monoclonal gammopathy seen in the Dysproteinemia Clinic are routinely screened for symptoms and signs of amyloidosis. Congo red staining of the bone marrow is not routinely done unless there is clinical suspicion of amyloidosis. For the purpose of this study, the medical records of these patients were reviewed to discern whether there had been coexisting signs or symptoms suggestive for symptomatic $\mathrm{AL}$ at the time of diagnostic $\mathrm{BM}$ for $\mathrm{MM}$ or SMM. Permission to perform a retrospective chart review was granted by the Institutional Review Board of the Mayo Foundation in accordance with federal regulations.

During the same time period, there were 691 cases of AL diagnosed at Mayo within 30 days of their initial diagnosis.
Of them, $39(5.6 \%)$ had more than $10 \%$ bone marrow plasmacytosis, consistent with coexisting multiple myeloma. Of these, only $18(46.1 \%$ of patients with $>10 \%$ of BM plasma cells) or $2.6 \%$ of the entire $\mathrm{AL}$ population had symptoms consistent with myeloma, i.e., bone lesions, anemia $(\mathrm{Hb}<11 \mathrm{~g} / \mathrm{dL})$, calcium greater than $10.5 \mathrm{mg} / \mathrm{dL}$, or myeloma-related kidney disease, which was defined as a creatinine greater than $1.5 \mathrm{mg} / \mathrm{dL}$ and a $24-\mathrm{h}$ urinary Mprotein to 24-h urinary total protein ratio of greater than 0.5 .

\section{Diagnosis of asymptomatic AL}

Bone marrow biopsies were reviewed and examined for Congo red staining. A patient was considered to have amyloid if the stain was positive, and apple-green birefringence was found on polarized microscopy. Posterior superior iliac crest was the preferred biopsy site, and the specimens were obtained with a modified Jamshidi needle as an outpatient procedure. Following decalcification, the bone marrows were paraffin embedded and H\&E stained, and Congo red stained sections were reviewed by a single author (WB). Amyloid deposits were detected by examination at $200 \times$ under polarized light. Only data from patients with an adequate follow-up period were considered in the final analysis. In addition, serum samples were obtained at diagnosis to determine LDH, $\beta 2$ microglobulin, albumin, calcium, phosphorus, creatinine, and liver function tests. Serum and urine electrophoresis and immunofixation, as well as bone marrow aspiration and bone X-ray studies, were taken at diagnosis.

\section{Statistical analysis}

Baseline differences between groups were assessed by the Chi-square test (Fisher's exact test) for categorical variables and univariate analysis of variance (ANOVA test) or Mann-Whitney $U$ statistic test for parametric and nonparametric analyses. Survival was estimated using the method of Kaplan and Meier.

\section{Results}

Overall characteristics of MM and SMM patients

Of the 1,180 patients with a diagnosis of MM (959) or SMM (221) seen at Mayo Clinic within 30 days of their diagnosis, biopsies were available from 144 (F 59, M 84). Among the patients evaluated, 77 had a diagnosis of MM and 67 of SM. Characteristics of MM and SM patients at time of diagnosis are reported in Table 1. One hundred thirty-six (95.8\%) patients had a positive serum M-spike before diagnostic BM. Median serum M-spike at the time 
Table 1 Baseline patients' characteristics

\begin{tabular}{llr}
\hline Characteristics & MM population $(n=77)$ & SM population $(n=67)$ \\
\hline Median months from biopsy to last follow-up (range) & $65(10.4-169.2)$ & $64.6(0-210)$ \\
Such fatigue (\%) & $13(16.8)$ & $5(7.4)$ \\
Pain (\%) & $42(54.5)$ & $1(1.5)$ \\
Paresthesia (\%) & $1(1.3)$ & $3(4.4)$ \\
Bleeding (\%) & 0 & $1(1.5)$ \\
Edema (\%) & 0 & $1(1.5)$ \\
Dyspnea (\%) & $6(7.8)$ & $1(1.5)$ \\
Median age, years (range) & $55.5(33-70)$ & $63(26-84)$ \\
Hemoglobin $(\mathrm{g} / \mathrm{dL})$, median (range) & $11.0(7.6-15.3)$ & $12.5(7.6-14.8)$ \\
Platelets $\left(\times 10^{9} / \mathrm{L}\right)$, median (range) & $222.0(8-399)$ & $224.0(65-786)$ \\
Serum protein $(\mathrm{g} / \mathrm{dL})$, median (range) & $8.0(6-16.1)$ & $8.4(6.3-11.8)$ \\
Urine protein $(\mathrm{g} / 24$ h) & $0.13(0.01-10.7)$ & $0.1(0.01-2.7)$ \\
Serum albumin, median (range) & $3.64(2.3-5.7)$ & $3.60(2.3-4.7)$ \\
Alkaline phosphate $(\mathrm{U} / \mathrm{L})$, median (range) & $175.5(53-1199)$ & $155.0(62-480)$ \\
Creatinine $(\mathrm{mg} / \mathrm{dL})$, median (range) & $1.1(0.7-2.2)$ & $1.1(0.9-6.6)$ \\
\hline
\end{tabular}

$B M$ bone marrow, $F U$ follow-up

of diagnostic BM biopsy was 2.93 (range 0.6-9.5). At a median follow-up of living patients of 76.2 months (range 0-215.9), 46 (31.9\%) patients were alive, 93 (64.5\%) dead, and $5(3.4 \%)$ lost to follow-up. The overall median survival was 70.4 months.

At presentation, SMM patients were older, less anemic, had higher creatinine and urine protein, but lower urine Mspike levels than MM patients. These last patients were more likely to have fatigue and pain, while SMM patients had more frequently paresthesia (Table 1).

Patients with asymptomatic amyloid deposit at presentation

Amyloid was retrospectively detected in the index bone marrow biopsy of two of the 144 cases $(1.38 \%, 95 \%$ CI -0.6 to 3.2), one in MM, and one in SMM group, respectively. The first (OE, female, 66 years old) was diagnosed having MM; at presentation, she had moderate anemia ( $\mathrm{Hg} 9.4 \mathrm{~g} / \mathrm{l})$, pain, multiple fractures but not hypercalcemia, renal, or liver tests alteration. No measurable M-spike was identified on electrophoresis, but there was a monoclonal kappa light chain detectable in the serum by immunofixation. Urine protein electrophoresis showed a total urine protein of $280 \mathrm{mg}$ with an M-spike of $249 \mathrm{mg} / 24 \mathrm{~h}$ that was confirmed to be kappa light chain. Quantitative immunoglobulins were not done. The bone marrow biopsy revealed plasma cells comprising $30 \%$ of the total cellularity. Beta-2-microglobulin was elevated at $2.28 \mu \mathrm{g} / \mathrm{mL}$. Cytogenetic studies by FISH did not show IGH translocation, monosomy, or deletion 13 , deletion of p53 or trisomy 3, 7, 9, 11, 13, 15, or 17. Cytogenetics studies of BM showed a normal karyotype (46, XX). The second patient (RW, male, 77 years old) was diagnosed with SMM (IgG-K).
He had a history of weight loss and severe chronic obstructive pulmonary disease. At presentation, physical exam did not reveal any abnormalities. The BM biopsy revealed plasma cells up to $25 \%$ of the total cellularity; $\beta 2$-microglobulin was $1.52 \mu \mathrm{g} / \mathrm{mL}$. Bone survey was negative for metastases or myeloma. Clinical and laboratory characteristics are listed in Table 2. After more than 7 years of follow-up, neither of these patients had or developed during their disease signs or symptoms suggestive for organ involvement by amyloid.

Patients without bone marrow amyloid deposition at presentation but subsequent systemic amyloidosis

There was one patient (RJ, male, 51 years old) who was diagnosed having MGUS (IgG- $\lambda$ ) in 1992 followed by SMM in 1993. Clinical and laboratory characteristics at presentation are listed in Table 2. At the presentation, physical examination was normal. He did not have weakness, fatigue, bleeding, or increased bruising, not bone pain, change in his voice or tongue, steatorrhea, paresthesia, claudication, or weight loss. He developed symptomatic AL after 72.6 months (February 1999) from initial diagnosis of SMM. He was treated with melphalan/prednisone. At the time of last contact in 2002, he considered himself to be in good health.

\section{Discussion}

Rate and clinical impact of asymp-amyloid in patients with $\mathrm{MM}$ or SMM is uncertain. While asymptomatic amyloid 
Table 2 Characteristics of two patients with detection of amyloid deposit at presentation and one patient without amyloid deposit but subsequent systemic amyloidosis

\begin{tabular}{|c|c|c|c|}
\hline \multirow[t]{2}{*}{$\begin{array}{l}\text { Clinical/laboratory } \\
\text { characteristics at diagnosis }\end{array}$} & \multicolumn{2}{|c|}{$\begin{array}{l}\text { Patients with amyloid } \\
\text { deposit at presentation }\end{array}$} & \multirow[t]{2}{*}{$\begin{array}{l}\text { Patient without amyloid deposit } \\
\text { at presentation but subsequent } \mathrm{AL}\end{array}$} \\
\hline & $\begin{array}{l}\text { MM } \\
\text { patient }\end{array}$ & $\begin{array}{l}\text { SM } \\
\text { patient }\end{array}$ & \\
\hline Months from biopsy to last follow-up & 86.4 & 87.6 & 72.6 \\
\hline Age (year) & 66 & 77 & 51 \\
\hline $\mathrm{Hg}(\mathrm{g} / \mathrm{dL})$ & 9.4 & 13 & 12.3 \\
\hline Platelets $\left(n \times 10^{9} / \mathrm{L}\right)$ & 328 & 201 & 446 \\
\hline Pro-thrombin time (s) & 10.1 & NA & NA \\
\hline Serum protein $(g / d L)$ & NA & 7.4 & 6.9 \\
\hline Urine protein $(\mathrm{mg} / \mathrm{dL})$ & 280 & 8 & 13 \\
\hline Serum Albumin (g/dL) & 2.9 & 3.6 & 3.5 \\
\hline Alkaline phosphate (U/L) & NA & 145 & 152 \\
\hline Creatinine $(\mathrm{mg} / 100 \mathrm{~mL})$ & 0.8 & 1.1 & 0.9 \\
\hline B2-microglobulin $(\mu \mathrm{g} / \mathrm{mL})$ & 2.28 & 1.56 & 1.5 \\
\hline C-reactive protein level (mg/dL) & NA & NA & 0.59 \\
\hline Serum light chain isotype & Kappa & Kappa & Lambda \\
\hline
\end{tabular}

deposits have been reported to coexist at the time of MM diagnosis in up to $30 \%$ of patients $[1,5]$, less information is available in patients with established MM or SMM who are seen at a facility at which an extensive amyloid directed review of systems and signs is the norm. This issue is topical since unrecognized $\mathrm{AL}$ can be associated with significant mortality and morbidity, and its early detection could change the management of MM and SMM patients.

We found that only $1.4 \%$ of patients with retrospective Congo red staining of their bone marrow had amyloid present on their presenting bone marrow. This may be a limitation of the present investigation, since it is well known that the sensitivity of bone marrow biopsy is only about $50 \%$ in comparison to that of abdominal fat aspirate with a sensitivity of about $80 \%$ of confirmed amyloidosis [1, 11]. It is possible that our approach yielded a low estimate. For example, the one patient, whose initial bone marrow was negative for amyloidosis but who was diagnosed with AL 6 years later, might have had a positive fat aspirate at the time of his earlier bone marrow; if so, he would have had very indolent amyloidosis.

Our data, which may appear to conflict with prior reports, would suggest that a careful amyloid-specific review at the time of diagnosis of SMM or MM misses only $1-2 \%$ of cases even with a 6.4 person-years of followup and therefore abrogates the need to do routine amyloid staining on all cases of SMM or MM. It also supports the Mayo Clinic practice of having a low threshold for amyloidosis screening if non-specific symptoms are present in a patient with a monoclonal gammopathy.

Table 3 puts our findings into the context of prior reports. One of the first reports addressing this question was by Desikan et al. [6], who prospectively evaluated fat pad aspirate and BM biopsy for amyloid deposit in a cohort of 84 MM patients. Amyloid was detected in $38 \%$ of patients; fat pad aspirate was positive in $25 \%$ and $\mathrm{BM}$ in $10 \%$ of cases and other organ sites in seven patients. MM or SMM patients did not differ in disease characteristics, and particularly, no $\lambda$ predominance was found. The median overall and event-free survival of the entire cohort is $66+$ and 52 months respectively; the median overall (59+ months vs $66+$ months, $p=0.9)$ and event-free survival $(59+$ vs $52+$ months, $p=0.9)$ were similar in cohort with and without amyloid, respectively. These authors concluded, therefore, that although the incidence of light chain asymptomatic AL in MM patients is high, it does not influence the outcome of these patients treated with autologous transplantation.

Vela-Ojeda et al. evaluated 201 consecutive patients with de novo MM in whom a fat pad biopsy needle aspiration (stained with Congo red) was performed [8]. Overall, 68 patients (34\%) were diagnosed having concomitant AL; 16 (8\%) had symptoms consistent with amyloidosis at the time of $\mathrm{MM}$ diagnosis, while in $52(25.8 \%)$, fat pad aspiration was useful to detect incidental asymptomatic AL. The median follow-up was 36 months (range 12-199). Among patients without AL at the time of diagnostic BM for MM, $69 \%$ of them became symptomatic for AL after a median follow-up of 14 months (range 6 to 30). Even excluding 16 patients with obvious symptoms of AL at diagnosis, overall survival was worse in patients who developed late AL (13 vs 64 months of those without AL). Cox regression model for overall survival detected three variables having independent prognostic significance: the presence of AL (RR $3.4, p<0.004$ ), serum albumin levels $<3.5 \mathrm{~g} / \mathrm{dL}$ (RR 3.2, $p<$ 
Table 3 Studies considering relationship between asymptomatic AL in myeloma patients

\begin{tabular}{|c|c|c|c|c|c|c|c|}
\hline Author [ref] & $\begin{array}{l}\text { MM } \\
\text { cases, } n\end{array}$ & $\begin{array}{l}\text { AL cases, } \\
n(\%)\end{array}$ & $\begin{array}{l}\mathrm{AL} \\
\text { cases, } n\end{array}$ & $\begin{array}{l}\text { MM case, } \\
n(\%)\end{array}$ & Tissues stained for amyloid & $\begin{array}{l}\text { Prognostic } \\
\text { factor }\end{array}$ & \\
\hline \multicolumn{8}{|c|}{ MM cases with incidental amyloidosis } \\
\hline $\begin{array}{c}\text { Desikan } \\
{[6]}\end{array}$ & 84 & $32(30.8)$ & & & $\begin{array}{l}\text { Subcutaneous abdominal fat } \\
\text { aspirate (SAFA) in } 25 \text {, bone } \\
\text { marrow in } 8 \text {, and other organ } \\
\text { sites in } 7 \text { patients }\end{array}$ & No & \\
\hline $\begin{array}{l}\text { Vela-Ojeda } \\
{[8]}\end{array}$ & 201 & $68(34)^{\mathrm{a}}$ & & & SAFA & Yes & \\
\hline $\begin{array}{l}\text { Petruziello } \\
\text { [12] }\end{array}$ & 166 & $67(40.3)$ & & & $\begin{array}{l}\text { Cytological techniques in } \\
\text { bone marrow smears }\end{array}$ & No & \\
\hline $\begin{array}{l}\text { Present study } \\
\text { MM }\end{array}$ & 77 & $1(1.3)$ & & & Bone marrow & No & \\
\hline $\begin{array}{l}\text { Present study } \\
\text { SMM }\end{array}$ & 67 & $1(1.5)$ & & & Bone marrow & No & \\
\hline \multicolumn{8}{|c|}{ AL cases with asymptomatic MM } \\
\hline $\begin{array}{l}\text { Kyle } \\
\text { [2] } \\
\text { AL cases with }\end{array}$ & symptor & natic MM & 474 & & & & \\
\hline $\begin{array}{l}\text { Rajkumar } \\
{[4]}\end{array}$ & & & 1,596 & $6(0.3)$ & Biopsy-proven AL & & $\begin{array}{l}\text { No laboratory or clinical evidence of MM } \\
\text { at the diagnosis of AL. MM developed } \\
10-81 \text { months hereafter }\end{array}$ \\
\hline
\end{tabular}

${ }^{\mathrm{a}}$ Sixteen were symptomatic for $\mathrm{AL}$

0.005 ), and patients not achieving complete remission or very good partial remission ( RR 2.9, $p<0.02$ ). The authors then concluded that MM-associated AL represents a poorer prognosis of disease even in the absence of symptoms at diagnosis, and this specific association may be considered as an independent high-risk prognostic factor. Thus, authors concluded that routine study of peri-umbilical fat pad tissue should be mandatory in all patients with MM.

Petruzziello et al. (2009) evaluated retrospectively the bone marrow smears of 166 unselected patients affected by MM (126 at diagnosis and 40 after treatment); no data were reported about the presence of signs or symptoms suggestive for concomitant systemic AL [12]. All samples were stained with Congo red and studied by transmission and birefringence microscopy. Both focal and diffuse involvements were considered positive. Overall, 67 patients were positive for AL, and 99 were negative to Congo red and apple-green birefringence. In particular, 51 of the 126 patients studied at diagnosis and 16 of the 40 patients with advanced disease were positive. Authors failed to find correlations between bone marrow amyloid deposits and immunoglobulin type, disease stage, plasma cells percentage, hemoglobin, calcium, creatinine, albumin, or $\beta 2$-microglobulin.

Although the main objective of our investigation was to evaluate the rate of asymp-amyloid at the time of first $\mathrm{BM}$, we were able to look at the clinical outcome of our cohort who had a median follow-up of 6 years. Neither of the patients who were retrospectively found to have amyloid in their index bone marrows developed symptomatic $\mathrm{AL}$, and their survival did not differ from the other patients. Among Congo red negative patients, we detected one (SMM patient) who developed symptomatic AL during a follow-up of 72.6 months. He had serum $\lambda$ light chain, an isotype frequently reported in patients with AL [13].

One potential caveat in studies looking at the detection of asymp-amyloid relies on patients' selection. In fact, $\mathrm{AL}$ requires a high index of suspicion, and most of $\mathrm{AL}$ cases are often misdiagnosed with other PCDs, mainly with MM (The UK Myeloma Forum AL Amyloidosis Guidelines Working Group, 2004). It is not unlikely that the true occurrence of asymptomatic AL has been previously overestimated. Because of the high level of suspicion for amyloid at our Amyloid Center, we are confident that all patients evaluated did not have (even subclinical) AL at the time of diagnostic BM for MM or SM. Therefore, evaluation of adequate specimens in experienced laboratories is necessary to maintain high diagnostic sensitivity and specificity.

In conclusion, we evaluated rates and clinical significance of amyloid deposit at the time of first diagnostic BM biopsy in newly diagnosed MM and SMM. Our findings show that fewer than $2 \%$ of patients in both groups have asymp-amyloid, thus discouraging any specific search for amyloid deposit when careful evaluation of clinical presentation and patient's history are not suggestive for systemic AL. 


\section{References}

1. Gertz MA, Lacy MQ, Dispenzieri A (1999) Amyloidosis. Hematol Oncol Clin North Am 13:1211-1233

2. Kyle RA, Gertz MA (1995) Primary systemic amyloidosis: clinical and laboratory features in 474 cases. Semin Hematol 32:45-59

3. Rajkumar SV, Dispenzieri A, Kyle RA (2006) Monoclonal gammopathy of undetermined significance, Waldenström macroglobulinemia, AL amyloidosis, and related plasma cell disorders: diagnosis and treatment. Mayo Clin Proc 81(5):693-703

4. Rajkumar SV, Gertz MA, Kyle RA (1998) Primary systemic amyloidosis with delayed progression to multiple myeloma. Cancer 82:1501-1505

5. Kyle RA (1995) Multiple myeloma and other plasma cell disorders. In: Hoffman R, Benz EJ, Shattil SJ, Cohen FB, LE HJ S (eds) Hematology, basic principles and practice, 2nd edn. Churchill Livingstone, New York, pp 1354-1374

6. Desikan KR, Dhodapkar MV, Hough A et al (1997) Incidence and impact of light chain associated (AL) amyloidosis on the prognosis of patients with multiple myeloma treated with autologous transplantation. Leuk Lymphoma 27:315-319
7. Bahlis NJ, Lazarus HM (2006) Multiple myeloma-associated AL amyloidosis: is a distinctive therapeutic approach warranted? Bone Marrow Transplant 38(1):7-15

8. Vela-Ojeda J, García-Ruiz Esparza MA, Padilla-González Y et al (2009) Multiple myeloma-associated amyloidosis is an independent high-risk prognostic factor. Ann Hematol 88 (1):59-66

9. The UK Myeloma Forum AL Amyloidosis Guidelines Working Group (2004) Guidelines on the diagnosis and management of AL amyloidosis. Br J Haematol 125:681-700

10. Kyle RA, Remstein ED, Therneau TM et al (2007) Clinical course and prognosis of smoldering (asymptomatic) multiple myeloma. N Engl J Med 356(25):2582-2590

11. Duston MA, Skinner M, Shraham T et al (1987) Diagnosis of amyloidosis by abdominal fat aspiration: analysis of 4 years experience. Am J Med 82:412-414

12. Petruzziello F, Zeppa P, Catalano L et al (2010) Amyloid in bone marrow smears of patients affected by multiple myeloma. Ann Hematol 89(5):469-474

13. Solomon A, Weiss DT, Kattine AA et al (1991) Nephrotoxic potential of Bence Jones proteins. N Engl J Med 324(26):18451851 